\title{
Site-specific fibroblasts regulate site-specific inflammatory niche formation in gastric cancer
}

\author{
Anna Abe ${ }^{1,2} \cdot$ Akiko Kawano Nagatsuma $^{2}$ - Youichi Higuchi ${ }^{1,2} \cdot$ Yuka Nakamura $^{2}$. \\ Kazuyoshi Yanagihara ${ }^{2} \cdot$ Atsushi Ochiai ${ }^{1,2}$
}

Received: 27 September 2015 / Accepted: 28 November 2015/Published online: 22 December 2015

(c) The International Gastric Cancer Association and The Japanese Gastric Cancer Association 2015

\begin{abstract}
Background Fibroblasts are the commonest type of cancer stromal cells. Inflammation occurs in cancer tissue, and the inflammatory process has been suggested to be caused by interactions between immune cells and cancer cells. In this study, we clarified that site-specific fibroblasts regulate the formation of a site-specific inflammatory niche according to the depth of gastric cancer cell invasion.
\end{abstract}

Akiko Kawno Nagatsuma is now on childcare leave. However, she has read this article and has agreed to its submission.

Electronic supplementary material The online version of this article (doi:10.1007/s10120-015-0584-y) contains supplementary material, which is available to authorized users.

Atsushi Ochiai

aochiai@east.ncc.go.jp

Anna Abe

anabe@east.ncc.go.jp

Akiko Kawano Nagatsuma

akawano@east.ncc.go.jp

Youichi Higuchi

yohiguch@east.ncc.go.jp

Yuka Nakamura

yukanaka@east.ncc.go.jp

Kazuyoshi Yanagihara

kyanagih@east.ncc.go.jp

1 Laboratory of Cancer Biology, Department of Integrated Bioscience, Graduate School of Frontier Science, University of Tokyo, Kashiwa, Chiba, Japan

2 Pathology Division, Exploratory Oncology Research and Clinical Trial Center, Research Center for Innovative Oncology, National Cancer Center Hospital East, 6-5-1 Kashiwanoha, Kashiwa, Chiba 277-8577, Japan
Methods Immunohistochemistry was performed with paraffin-embedded tissues. The numbers of immune cells and the fibroblast area were calculated according to the cancer depth. The gene expression patterns of submucosal fibroblasts and subperitoneal fibroblasts stimulated with HSC44PE-conditioned medium were analyzed with a microarray. To examine the effects on the cancer microenvironment of differences in gene expressions between HSC44PE-stimulated submucosal fibroblasts and subperitoneal fibroblasts, assays of HSC44PE proliferation, $\mathrm{T}$ cell migration, and M2-like macrophage differentiation were performed.

Results The distributions of immune cells differed between the submucosal layer and the subserosal layer. The number of M2 macrophages was significantly higher and the fibroblast area was significantly larger in the subserosal layer compared with the submucosal layer. High expression levels of IL1B, TNFSF15, and CCL13 were observed in HSC44PE-stimulated submucosal fibroblasts, and higher expression levels of TGFB2, CSF1, CCL8, and CXCL5 were found in HSC44PE-stimulated subperitoneal fibroblasts. HSC44PE-stimulated subperitoneal fibroblast medium promoted the differentiation of monocytes into M2like macrophages, whereas HSC44PE-stimulated submucosal fibroblasts significantly induced the migration of Jurkat cells and the growth of HSC44PE cells.

Conclusion The dynamic states of immune cells differ between the submucosal and subserosal layers in cancer tissues. Site-specific fibroblasts regulate site-specific inflammatory niche formation according to the depth of cancer cell invasion.

Keywords Inflammation - Gastric cancer - Immune cells · Fibroblasts - Inflammatory niche 


\section{Introduction}

Fibroblasts are among the commonest types of stromal cells in connective tissues and are essential for tissue homeostasis and wound healing in the process of inflammation [1]. Fibroblasts comprise a very heterogeneous population of cells, with each subpopulation exhibiting different pathophysiological functions [2]. We previously demonstrated in our laboratory that colon fibroblasts obtained from submucosal and subperitoneal tissues showed biological differences and differential gene expression [3]. In cancer tissues, whereas some cancerassociated fibroblasts promote cancer cell proliferation and migration, others suppress cancer cell growth.

Rudolf Virchow hypothesized that cancer arises from inflammatory sites, so-called sites of "lymphoreticular infiltration". Abundant evidence has been collected to suggest that infection and inflammatory disease serve as triggers for the development of various cancers $[4,5]$. In addition to fibroblasts, cancer stroma also contains new blood vessels and connective tissue. In 1986, Dvorak showed that wound healing and tumor stroma formation share many important properties $[5,6]$ and defined cancers as wounds that do not heal [7]. Therefore, inflammation is an inherent pathophysiological process in cancer tissues. Many reports have suggested that cancer-associated inflammation is caused by interactions between immune cells and cancer cells.

Inflammation has been reported to be closely involved in the initiation, progression, and metastasis of gastric cancer [8]. For example, persistent infection with Helicobacter pylori leads to the stimulation of immune cells by bacterial toxins, resulting in the production of a wide variety of inflammatory factors, including tumor necrosis factor (TNF)- $\alpha$, interleukin (IL)-1, IL-6, and IL-8. These factors cause inflammation and promote the development of gastric cancer and the infiltration of lymphocytes $[9,10]$. Moreover, IL-6 is produced mainly by macrophages and lymphocytes under the regulation of the nuclear factor $\kappa \mathrm{B}$ signaling pathway. IL-6 is also linked to signal transducer and activator of transcription 3 and exerts multiple actions, including growth-promoting and antiapoptotic actions on cancer cells. Therefore, the elevation of the serum level of IL-6 has been proposed as a poor prognostic factor for recurrence and overall survival in gastric cancer patients [11,12]. Consistent with these reports, a number of other reports have also suggested that the key features of cancer-associated inflammation are the production of cytokines by immune cells and the interactions between cancer cells and immune cells [13, 14].

On the basis of the observation that fibroblasts are among the commonest cell types within the stroma, it has been suggested that they may play important roles in cancer-associated inflammation. In addition to stimulating cell adhesion and inducing leucocyte activation during the process of inflammation, inflammatory fibroblasts from the rheumatoid synovium have also been shown to promote the onward migration of $\mathrm{T}$ cells through the endothelial cell layer in co-culture models [15]. In pancreatic cancer, transgenic mice with a deficiency of alpha smooth muscle actin ( $\alpha$-SMA) myofibroblasts showed immune suppression as a result of an increase in the number of regulatory $\mathrm{T}$ cells [16]. We also reported that HSC44PE-conditioned medium induced the expression of mainly IL-8 in fibroblasts [17]. Fibroblasts are very heterogeneous and show biological differences between the submucosal layer and the subserosal layer in cancer tissue. Additionally, fibroblasts have the ability to induce the migration of immune cells to an inflammatory niche in cancer tissue.

These reports suggest that in gastric cancer, site-specific fibroblasts regulate site-specific inflammatory niche formation, according to the depth of the invasion of the gastric cancer cells. In this study, we focused on inflammatory niche formation according to the depth of the invasion of gastric cancer cells. We demonstrated that each type of fibroblast plays its own role in inflammatory niche formation in gastric cancer by comparing the character of stromal cells, such as immune cells and fibroblasts, with that of the submucosal layer and that of the subserosal layer.

\section{Materials and methods}

\section{Cell lines}

Two human gastric cancer cell lines were used in the study. MKN7 was obtained from Immuno-Biological Laboratories (Gunma, Japan) and HSC44PE was established previously [18]. MKN7 was maintained in Dulbecco's modified Eagle's medium (DMEM; Life Technologies, NM, USA) containing $10 \%$ heat-immobilized fetal bovine serum (FBS; Life Technologies) and supplemented with penicillin and streptomycin (Life Technologies). HSC44PE, Jurkat, and THP1 cells were maintained in RPMI 1640 medium (Life Technologies) containing $10 \%$ heat-immobilized FBS and supplemented with penicillin and streptomycin.

\section{Human primary fibroblast cells}

Noncancerous submucosal or subperitoneal tissues (at least $5 \mathrm{~cm}$ from the cancer lesion) were obtained from surgically resected specimens of human gastric cancer patients. The procedure for isolation of human primary fibroblast cells (submucosal gastric fibroblasts, SMFs; subperitoneal gastric fibroblasts, SPFs) has been reported previously [19]. 
The SMFs and SPFs were maintained in minimum essential medium $\alpha$ (Life Technologies) containing $10 \%$ heat-immobilized FBS and supplemented with penicillin and streptomycin. The study protocol was approved by the Institutional Review Board of the National Cancer Center Hospital East.

\section{Antibodies}

Mouse monoclonal antibodies against CD8, CD15, CD56, CD68, Ki67, and $\alpha$-SMA were obtained from Dako (Glostrup, Denmark). Mouse monoclonal antibodies against CD4 and CD20 were purchased from Abcam (Cambridge, UK). The mouse monoclonal antibody against CD204 was purchased from Trans Genic (Kumamoto, Japan). Horseradish peroxidase (HRP)-labeled speciesspecific whole antibody was also obtained from Dako.

\section{Immunohistochemistry}

Immunohistochemistry was performed with paraffin-embedded tissues obtained from 20 surgically resected specimens of human gastric cancer. The cancer had invaded the serosa in 20 specimens. Ten specimens exhibited up to moderate differentiation and were classified as differentiated subtype, and ten specimens were classified as poorly differentiated subtype. Most of the cancers had heterogeneous components, especially in terms of the differentiation status. Seven of 20 cases which consisted of an admixture of differentiated and poorly differentiated subtypes were classified into four cases with differentiated subtype and three cases with poorly differentiated subtype, respectively, according to their predominancy on the basis of the rules of the Japanese Classification of Gastric Carcinoma (14th edition, 2010).

After deparaffinization, the paraffin sections were immersed in the target retrieval solution at $95^{\circ} \mathrm{C}$ for $20 \mathrm{~min}$. To block endogenous peroxidase activity, the specimens were immersed in methanol containing $3 \%$ hydrogen peroxide. The samples were washed and incubated overnight at $4{ }^{\circ} \mathrm{C}$ with each of the antibodies. The sections were washed and incubated with EnVision+ System-HRP labeled polymer anti-mouse (Dako). The tissues were then incubated with diaminobenzidine peroxidase substrate for the indicated time. Nuclear counterstaining was performed with Mayer's hematoxylin solution (Muto Pure Chemicals, Tokyo, Japan).

\section{Immunohistochemical scoring of the immune cells, fibroblasts, and cancer cells}

Differential counts were performed for the submucosal layer, the muscularis propria, and the subserosal layer. The numbers of each type of immune cell [helper $\mathrm{T}$ cells $\left(\mathrm{CD}^{+}\right.$cells $)$, killer T cells $\left(\mathrm{CD}^{+}\right.$cells $)$, B cells $\left(\mathrm{CD} 20^{+}\right.$ cells), naïve $\mathrm{T}$ cells $\left(\mathrm{CD} 56^{+}\right.$cells), neutrophils $\left(\mathrm{CD} 15^{+}\right.$ cells), macrophages $\left(\mathrm{CD}^{+} 8^{+}\right.$cells), and M2 macrophages $\left(\mathrm{CD} 204^{+}\right.$cells $\left.)\right]$in the cancer tissues and noncancer tissues (at least $5 \mathrm{~cm}$ from the cancer lesion) were counted in five selected fields from each layer. The $\alpha$-SMA-positive fibroblast area per field was measured with AxioVision release 4.7 (Zeiss, Oberkochen, Germany) and was expressed as the percentage of the total area.

Random high-power fields were selected to count the number of nuclei showing positive staining for Ki67. One thousand nuclei were counted, and the Ki67 immunoreactivity was expressed as the proportion of Ki67-positive cancer cells among 1000 cancer cells [20].

\section{Collection of gastric cancer cell conditioned medium}

On day 0 , gastric cancer cells $\left(5.0 \times 10^{6}\right.$ per dish) were incubated in DMEM containing $10 \%$ FBS. The cultureconditioned medium was replaced with DMEM not containing FBS on day 2 and was collected and filtered with a vacuum filtration system (Millipore, Billerica, MA, USA). The filtrates were dispensed in 1-mL aliquots into each tube on day 3 . The collected specimens were stored at $-80{ }^{\circ} \mathrm{C}$.

\section{Collection of HSC44PE-stimulated SMF medium and HSC44PE-stimulated SPF medium}

On day $0, \mathrm{SMFs}$ and SPFs $\left(5.0 \times 10^{5}\right.$ per dish $)$ were incubated in DMEM containing $10 \%$ FBS. HSC44PE-conditioned medium was added to the SMFs or SPFs on day 2. On day 3, the culture-conditioned medium were replaced with DMEM not containing FBS, and the conditioned medium (HSC44PE-stimulated SMF medium, HSC44PE-stimulated SPF medium) was collected and filtered with a vacuum filtration system (Millipore, Billerica, MA, USA); the filtrates were dispensed in 1-mL aliquots into each tube on day 4.

\section{Stimulation with gastric-cancer-cell-conditioned medium}

On day 0 , the SMFs and SPFs $\left(5.0 \times 10^{5}\right.$ per dish) were incubated in DMEM containing $10 \%$ FBS. Gastric-cancercell-conditioned medium or DMEM not containing FBS was added to the SMFs or SPFs on day 2, followed by incubation for the indicated periods.

\section{Quantitative real-time reverse transcriptase polymerase chain reaction}

Each of the fibroblasts was incubated with DMEM or HSC44PE-conditioned medium, and the THP1 cells were 
incubated with HSC44PE-SMF/SPF medium for the designated times. The cells were washed with phosphatebuffered saline and suspended in 1-mL of TRIzol. Total RNA was purified by the TRIzol/RNeasy minicolumn protocol (QIAGEN), and complement ray DNA was synthesized with a PrimeScript ${ }^{\circledR}$ RT reagent kit (TaKaKa, Shiga, Japan). Quantitative real-time reverse transcriptase polymerase chain reaction (qRT-PCR) was performed with a LightCycler 48 (Roche, Basel, Switzerland). A quality check of all the total RNA samples was performed with an Agilent Bioanalyzer with an RNA 6000 Nano assay kit (Agilent Technology), and the RNA integrity of all the RNA samples was confirmed to be greater than 9.0.

\section{Microarrays}

To analyze the messenger RNA (mRNA) expression levels of about 47,000 transcripts and variants from 38,500 wellcharacterized human genes, GeneChip Human Genome U133 Plus 2 arrays (Affymetrix) containing 54,675 probe sets were used. Target complementary RNA was generated from $100 \mathrm{ng}$ of total RNA from each sample with a $3^{\prime}$ IVT Express kit (Affymetrix). The procedures for target hybridization, washing, and staining with signal amplification were undertaken according to the supplier's instructions. The arrays were scanned with a GeneChip Scanner 3000 (Affymetrix).

\section{Statistical analysis of the microarrays}

The gene expression data were analyzed with GX12.6 (Agilent Technologies). Raw data were summarized with the MAS5 algorithm (Affymetrix) and were normalized to log-transformed and median-centered data for the numerical analysis to permit gene selection. The differentially expressed probe sets used in the supervised hierarchical clustering were selected on the basis of $P<0.05$ and fold changes greater than 2.0. $P$ values were calculated by a one-way ANOVA with Benjamini and Hochberg multiple correction. For the hierarchical clustering, average linkage clustering with the Pearson correlation distance was performed.

\section{Functional analysis of the microarrays}

The predicted target genes were imported for functional analysis into DAVID version 6.7, a Web-based tool for annotation, visualization, and integrated discovery. We performed the biological process of Gene Ontology (GO) and pathway analysis on the mRNAs of genes showing differential expression between fibroblasts stimulated with cancer cells (MKN7 and HSC44PE) and those stimulated with DMEM. The statistical significance of the results of the GO and pathway analysis was determined on the basis of $P<0.01$.

\section{Migration assay}

Jurkat cell migration was assessed with a modified Boyden's chamber assay-that is, in Transwell cell culture chambers (Corning, Corning, NY, USA). Polycarbonate filters with $5-\mu \mathrm{m}$ pores were used to separate the upper and lower chambers. On day 0 , Jurkat cells $\left(1.0 \times 10^{6}\right.$ cells per dish) were incubated in DMEM containing $10 \%$ FBS. On day 1, DMEM not containing FBS was added to the Jurkat cells. Then, the Jurkat cells were added to the upper compartment of the chamber at a density of $1.0 \times 10^{7}$ cells per milliliter of DMEM not containing FBS and were incubated for $4 \mathrm{~h}$. The Jurkat cells were allowed to migrate toward the reagents (HSC44PE-SMF medium, HSC44PESPF medium) in the lower chamber. After the reaction, the cells that had migrated were collected from the bottom chamber and were counted with a hemocytometer [21]. The number of Jurkat cells in the positive control (DMEM containing FBS) was determined, and the rate of Jurkat cell migration was determined as the number of cells in the HSC44PE-stimulated fibroblast medium relative to that in DMEM.

\section{Cell viability assay}

Cell viability was measured with the 2-(2-methoxy-4-nitrophenyl)-3-(4-nitrophenyl)-5-(2,4-disulfophenyl)-2H-tetrazolium (WST-8) assay (Dojindo Laboratories) according to the manufacturer's instructions. HSC44PE cells $\left(5.0 \times 10^{6}\right.$ per dish $)$ were incubated in DMEM containing $10 \%$ FBS, penicillin, and streptomycin. After 2 days, HSC44PE cells were seeded in triplicate at $4.0 \times 10^{3}$ cells per well in a 96-well flat-bottomed culture plate containing HSC44PE-SPF medium, HSC44PE-SMF medium, DMEM-SPF medium, or DMEM-SMF medium. The WST-8 cell counting kit was added to each well, followed by incubation for $4 \mathrm{~h}$. Subsequently, the optical density was measured at $450 \mathrm{~nm}$.

\section{Differentiation of M2-like macrophages}

On day 0 , THP1 cells $\left(1.0 \times 10^{6}\right.$ cells per 10 -cm dish $)$ were incubated in DMEM containing $10 \%$ FBS. On day 2, DMEM not containing FBS was added to the THP1 cells. On day 3, the THP1 cells were treated for $48 \mathrm{~h}$ with HSC44PE-SMF medium or HSC44PE-SPF medium. Cells were collected and washed with phosphate-buffered saline and then suspended in $1 \mathrm{~mL}$ of TRIzol. 


\section{Statistical analysis}

The statistical significance of the difference between any two groups of interest was evaluated with the Student $t$ test. Differences were considered significant at $P<0.05$. For the results of the immunostainings, the error bars show the mean \pm standard deviation. For other results, the error bars show the mean \pm standard error. For the Ki67 labeling index, the statistical analysis was performed with the Wilcoxon signed-rank test.

\section{Results}

\section{Inflammatory niche formation in cancer tissue}

To investigate the differences in the inflammation niches according to the depth of invasion of gastric cancer, immunohistochemistry was performed with paraffin-embedded tissues obtained from 20 surgically resected specimens of human gastric cancer.

In the submucosal layer, the numbers of helper $\mathrm{T}$ cells, killer T cells, neutrophils, B cells, and macrophages, but not the number of naïve $\mathrm{T}$ cells (Fig. 1), were significantly higher in the cancer tissues than in the noncancer tissues. In the subserosal layer, the numbers of all the immune cell types except neutrophils and naïve $\mathrm{T}$ cells were significantly higher in the cancer tissues than in the noncancer tissues (Fig. 1). These results suggest that inflammation in cancer tissue is associated with infiltration by immune cells.

To examine whether the depth of cancer cell invasion influences the formation of the inflammatory niche, the numbers of each of the immune cell types and the area of the $\alpha$-SMA-positive fibroblasts were examined according to the depth of cancer cell invasion.

The numbers of each type of lymphocyte, including helper T cells, killer T cells, and B cells, were higher in the submucosal layer than in the subserosal layer (Figs. 1, S1). The number of neutrophils was also higher in the submucosal layer than in the subserosal layer (Figs. 1, S1). On the other hand, the number of macrophages was significantly higher in the subserosal layer than in the submucosal layer (Figs. 1, 2d). The area of $\alpha$-SMA-positive fibroblasts was larger in the subserosal layer than in the submucosal layer in cancer tissue (Fig. 2a, b). Additionally, $\alpha$-SMA-positive fibroblasts were not detected in noncancer tissues (Fig. 2b). $\alpha$-SMA-positive cells were recognized in smooth muscle fibers and vascular endothelial cells. The differences in the inflammatory niches between the submucosal layer and the subserosal layer were induced by stimulation with cancer cells. We found that only the number of macrophages was higher in the subserosal layer compared with the submucosal layer. Macrophages are classified into M1 macrophages, which are induced by nitric oxide and other molecules that promote cancer cell death, and M2 macrophages, which contribute to cancer progression and wound healing [22]. Therefore, the number of M2 macrophages was counted. Compared with the submucosal layer, the number and percentage of M2 macrophages were significantly higher in the subserosal layer (Fig. 2c, d).

The distributions of the immune cells were different between the submucosal layer and the subserosal layer. These results indicate that the inflammatory environment surrounding the cancer cells varies according to the depth of the invasion of the cancer cells, with the interaction between cancer cells and stromal cells regulating the formation of a specific inflammatory niche in each layer.

\section{Differential gene expression between SMFs and SPFs stimulated with HSC44PE-conditioned medium}

We examined whether differences in the stromal fibroblasts might underlie the difference in the inflammatory niches formed in the submucosal and subserosal layers in gastric cancer tissue, because fibroblasts are the most abundant stromal cells in cancer tissue [1].

To clarify whether fibroblasts were involved in the cancer-associated inflammation, we examined the gene expression in fibroblasts stimulated with gastric-cancercell-conditioned medium compared with the expression in fibroblasts stimulated with DMEM not containing FBS. MKN7 and HSC44PE cells were together considered as gastric cancer cells. Similarly, SMFs and SPFs were together considered as fibroblasts. The result of the GO analyses revealed that the fibroblasts stimulated with gastric-cancer-cell-conditioned medium were enriched in genes coding for cytokines and chemokines, which are factors involved in inflammation (Table 1). These results suggest that fibroblasts are closely involved in cancer-related inflammation.

We previously demonstrated that SMFs and SPFs stimulated with a colon-cancer-conditioned medium exhibit different gene expression patterns [3].

To examine whether the gene expression patterns of SMFs and SPFs stimulated with HSC44PE-conditioned medium differed, a microarray analysis was performed. Moreover, to confirm the mRNA expression of signature genes in the SMFs and SPFs, qRT-PCR was used. Genes encoding hormonal factors were extracted in a data analysis.

To consider the gene expression data together with the immunohistochemistry results, we extracted some sitespecific gene expression patterns in SMFs and SPFs stimulated with HSC44PE-conditioned medium (Fig. 3a). The results revealed higher expression levels of the genes encoding IL-1 $\beta$ (IL1B), TNF superfamily member 15 
Fig. 1 The numbers of each type of immune cell in cancer and noncancer tissues. Immunohistochemistry was performed with paraffinembedded tissues obtained from 20 surgically resected specimens of human gastric carcinoma. Differential counts were performed for the submucosal $(S M)$ layer, the muscularis propria $(M P)$, and the subserosal $(S S)$ layer. Helper $\mathrm{T}$ cells $\left(\mathrm{CD} 4^{+}\right.$cells), killer $\mathrm{T}$ cells $\left(\mathrm{CD}^{+}\right.$cells $), \mathrm{B}$ cells $\left(\mathrm{CD} 20^{+}\right.$cells), naïve $\mathrm{T}$ cells $\left(\mathrm{CD}^{+} 6^{+}\right.$cells), neutrophils $\left(\mathrm{CD} 15^{+}\right.$cells), and macrophages $\left(\mathrm{CD} 68^{+}\right.$cells $)$ were counted. $N D$ not determined

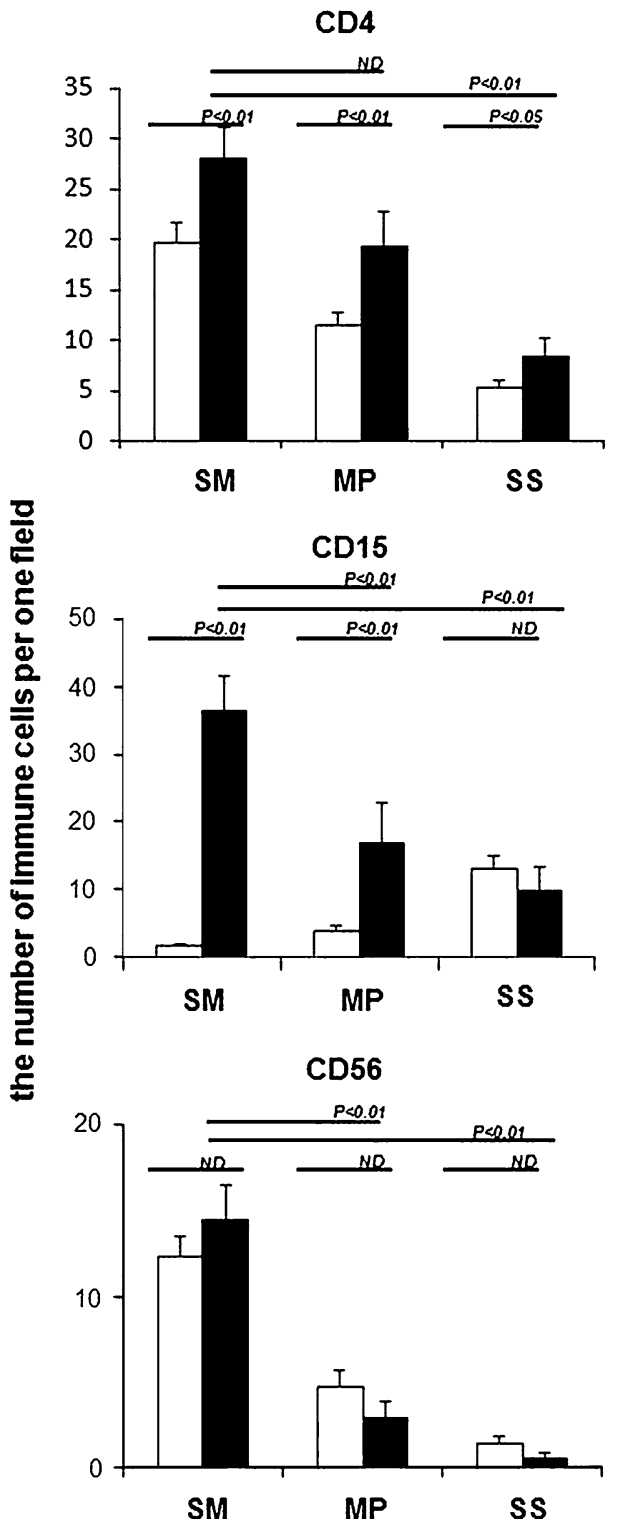

Non-cancer tissue

cancer tissue
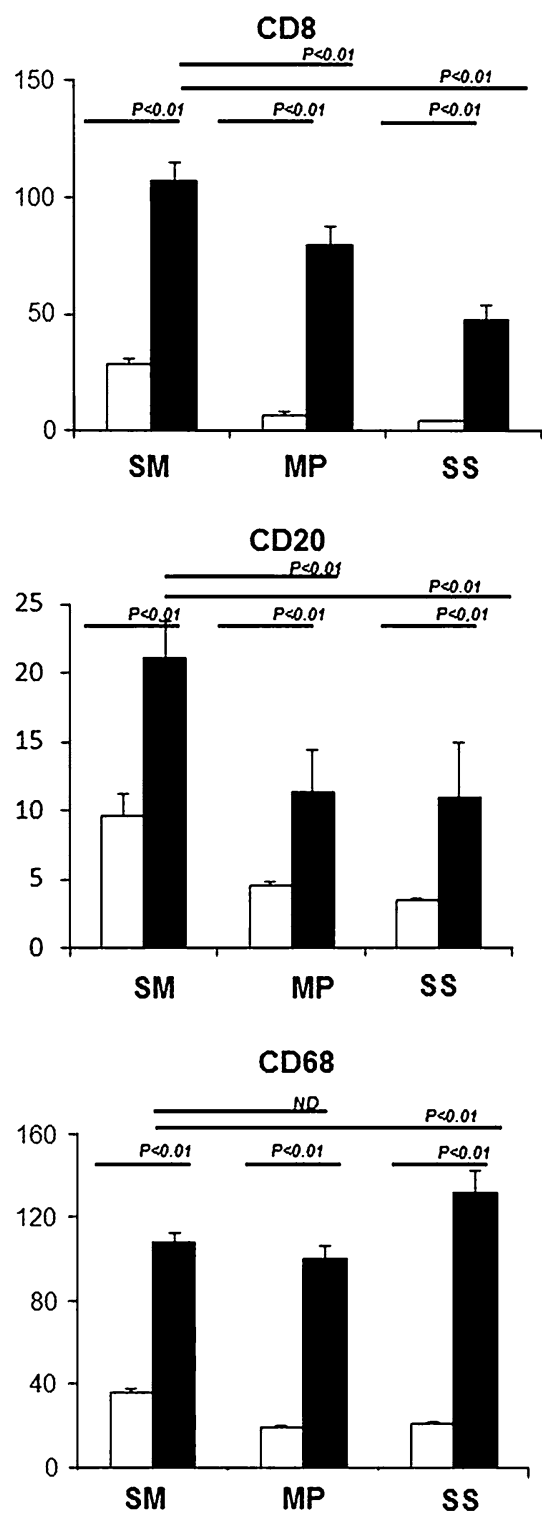

(TNFSF 15), and chemokine (C-C motif) ligand 13 (CCL13), which are involved in lymphocyte accumulation and cancer cell growth, in the HSC44PE-stimulated SMFs than in the HSC44PE-stimulated SPFs (Fig. 3a, b). On the other hand, higher expression levels of TGFB2, CSF1, CCL8, and CXCL5, which encode transforming growth factor $\beta_{2}$, colony stimulating factor 1 , chemokine $(\mathrm{C}-\mathrm{C}$ motif) ligand 8 , and chemokine $(\mathrm{C}-\mathrm{X}-\mathrm{C}$ motif) ligand 5 , which are involved in macrophage differentiation and tissue remodeling, were found in the HSC44PE-stimulated SPFs than in the HSC44PE-stimulated SMFs (Fig. 3a, c). These results suggest that the responses of fibroblasts to cancer cells differ depending on the site.
Effects of fibroblasts stimulated with cancer cells on the cancer microenvironment

To examine the effect of differences in the gene expression between SMFs and SPFs stimulated with HSC44PE-conditioned medium on the cancer microenvironment, assays for HSC44PE proliferation, T cell migration, and M2-like macrophage differentiation were performed.

The WST- 8 assay was performed to compare the effect of the HSC44PE-SMF medium and that of the HSC44PESPF medium on cancer cell growth. No significant differences were observed between the effect of the HSC44PESPF medium and the effect of DMEM-SPF medium. 
Fig. 2 Percentages of alpha smooth muscle actin (SMA)positive fibroblasts and number of CD204 ${ }^{+} \mathrm{M} 2$ macrophages. a Percentages of $\alpha$-SMApositive fibroblasts in the submucosal $(S M)$ layer, muscularis propria $(M P)$, and subserosal $(S S)$ layer in cancer tissue. The percentage was calculated as the ratio of the area of $\alpha$-SMA-positive fibroblasts to the total area $(n=20)$.

b Immunohistochemistry for $\alpha$ SMA-positive fibroblasts in the SM layer, MP, and SS layer in cancer and noncancer tissues $(n=20)$. Scale bar $50 \mu \mathrm{m}$. c Number and proportion of CD204 ${ }^{+}$M2 macrophages in the SM layer, MP, and SS layer. The proportion was calculated as the ratio of the number of CD204 ${ }^{+}$M2 macrophages to the number of CD68 ${ }^{+}$ macrophages $(n=20)$. d Immunohistochemistry for macrophages $\left(\mathrm{CD}^{+} 8^{+}\right.$cells) and M2 macrophages (CD204 ${ }^{+}$ cells) in the SM layer, MP, and SS layer $(n=20)$. Scale bar $50 \mu \mathrm{m}$ a

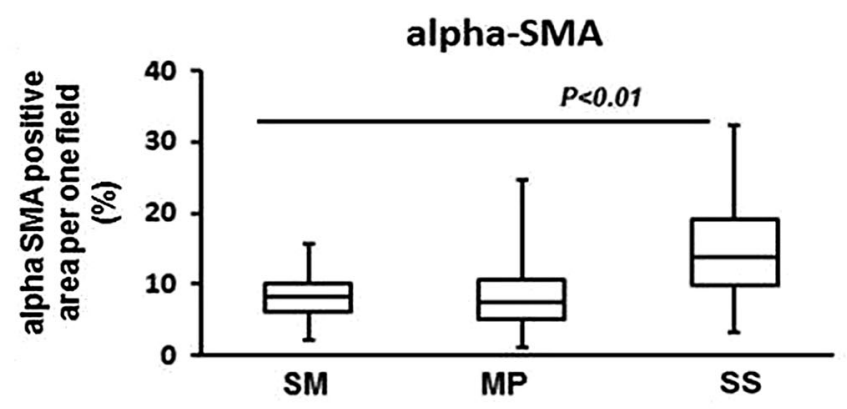

b
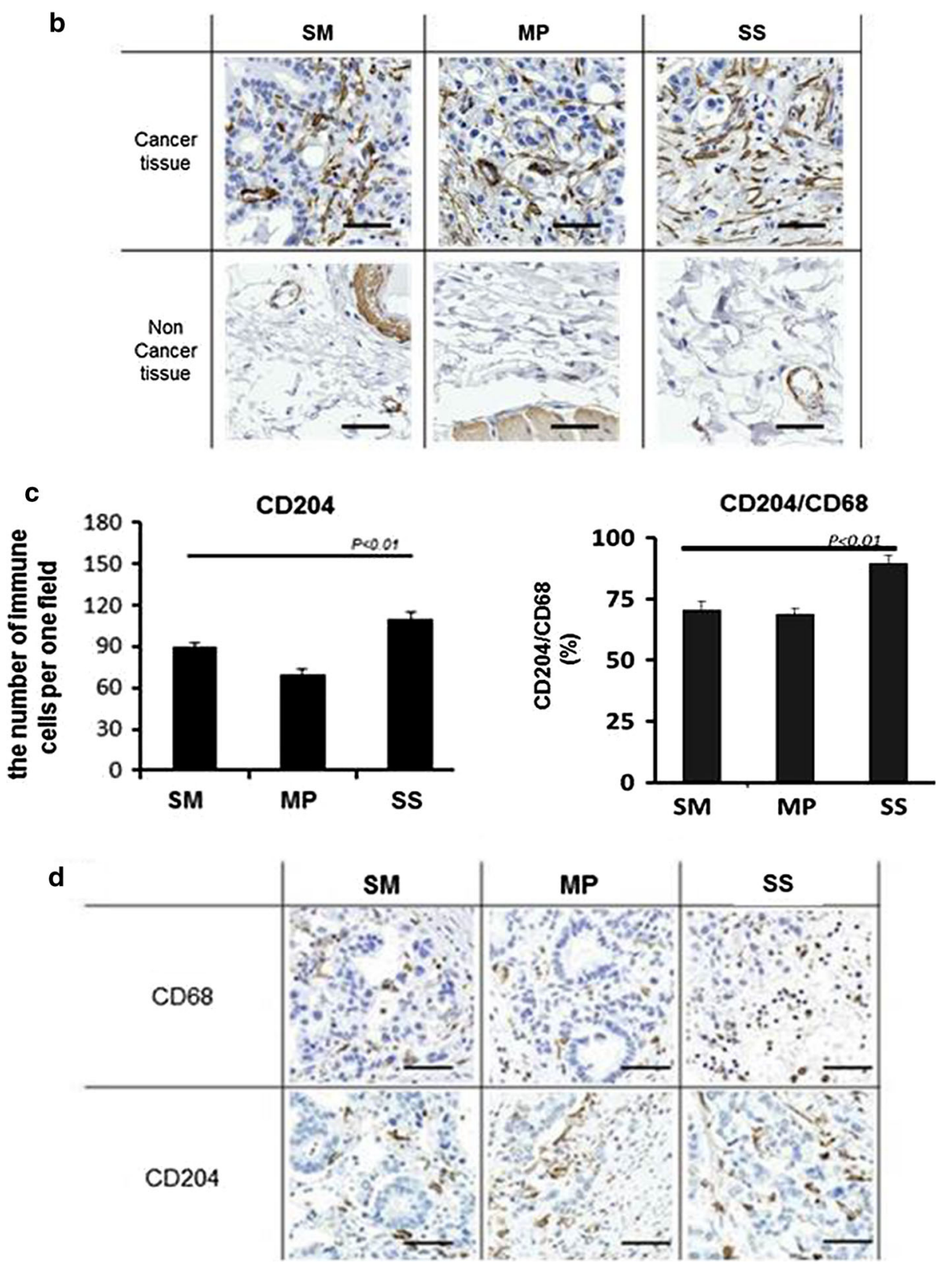

However, treatment with the HSC44PE-SMF medium promoted the growth of HSC44PE cells compared with treatment with the DMEM-SMF medium (Fig. 4c). Furthermore, in the 20 cases in which differential counts could be performed, we observed a significantly higher Ki67 labeling index in the submucosal layer than in the muscularis propria $(P<0.05)$ or the subserosal layer $(P<0.01)$. (Fig. 4a, b). These results suggest that the 
Table 1 Biological process of Gene Ontology $(G O)$ and pathway analysis of the messenger RNA expression of genes showing differential stimulated by cancer cells and Dulbecco's modified Eagle's medium expression between fibroblasts

\begin{tabular}{lll}
\hline Category & Term & $P$ \\
\hline GOTERM_MF_FAT & GO:0005125 cytokine activity & $9.58 \times 10^{-21}$ \\
GOTERM_MF_FAT & GO:0008009 chemokine activity & $9.63 \times 10^{-13}$ \\
GOTERM_MF_FAT & GO:0042379 chemokine receptor binding & $2.20 \times 10^{-12}$ \\
GOTERM_MF_FAT & GO:0008083 growth factor activity & $5.37 \times 10^{-8}$ \\
GOTERM_MF_FAT & GO:0005507 copper ion binding & $4.95 \times 10^{-6}$ \\
GOTERM_MF_FAT & GO:0046870 cadmium ion binding & $7.19 \times 10^{-6}$ \\
GOTERM_MF_FAT & GO:0005539 glycosaminoglycan binding & $3.458 \times 10^{-3}$ \\
GOTERM_MF_FAT & GO:0030246 carbohydrate binding & $4.041 \times 10^{-3}$ \\
\hline
\end{tabular}
SMF-HSC44PE of hormonal genes between submucosal gastric fibroblasts (SMFs) and subperitoneal gastric fibroblasts (SPFs) stimulated with HSC44PE medium. a Supervised clustering with probe sets showed significantly different expression patterns between SMFs and SPFs stimulated with HSC44PE medium. Fifty-six significant probe sets were selected on the basis of $P$ values less than 0.05 and fold change greater than 2.0 (one-way ANOVA). The color scale for the gene expression range is +2.0 to $-2.0 \log$ in $\log$ base 2 . The numbers are the patient identification numbers. $(n=3)$. b Messenger RNA expression levels of $I L 1 B$ and TNFSF 15 in fibroblasts stimulated with HSC44PE medium. Three independent experiments were performed in triplicate.

c Messenger RNA expression levels of TGFB2 and CSF1 in fibroblasts stimulated with HSC44PE medium. Three independent experiments were performed in triplicate a
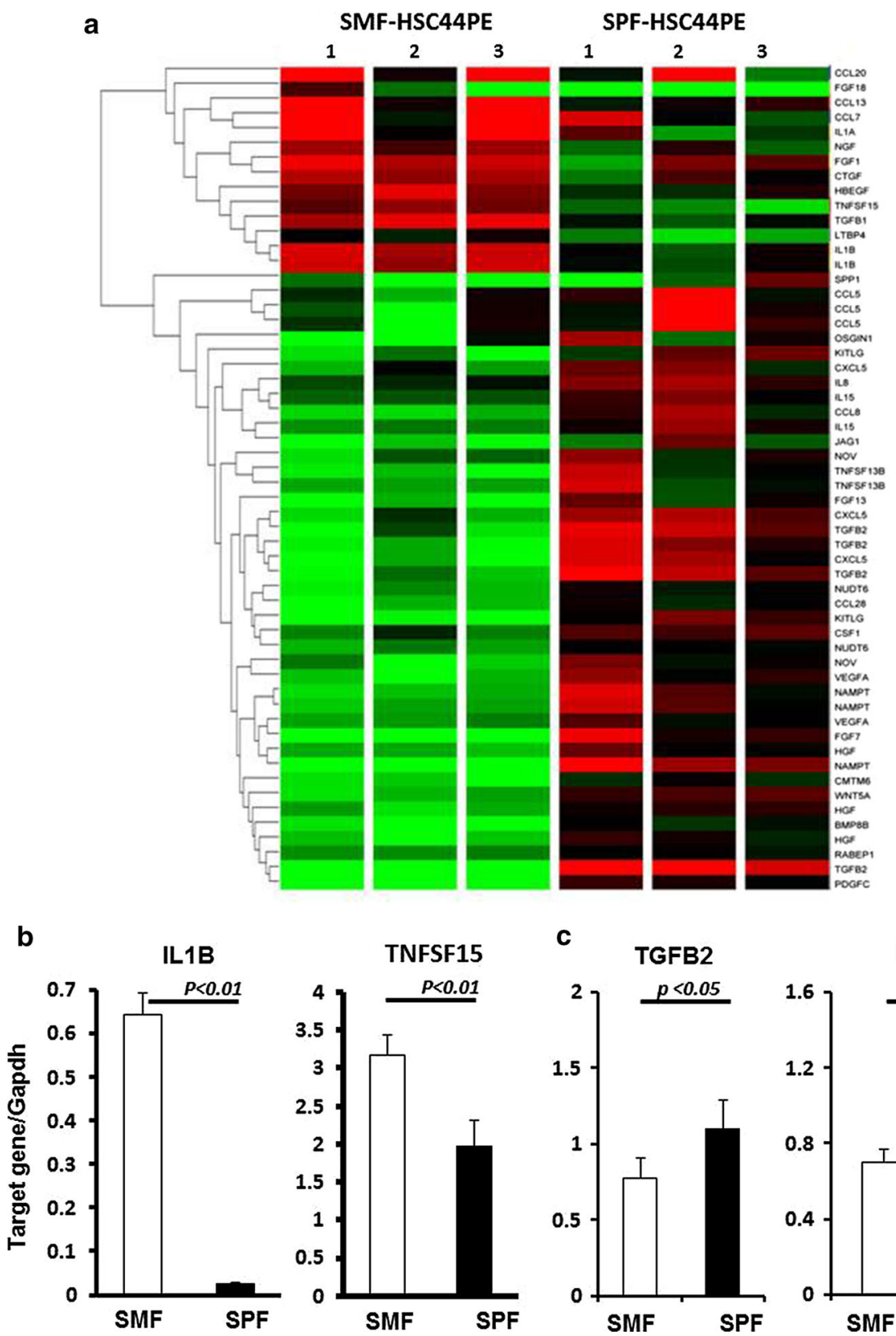

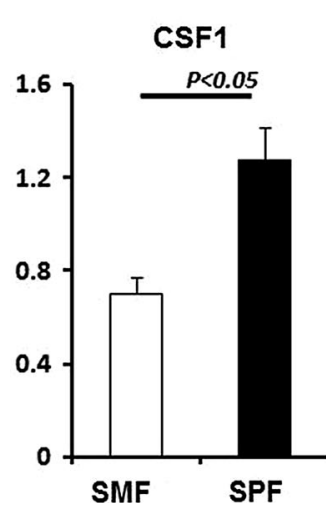


Fig. 4 Effects of fibroblasts stimulated with gastric cancer cells on cancer cell growth. a Scattergram showing Ki67 labeling index $(L I)$ in the submucosal $(S M)$ layer, muscularis propria $(M P)$, and subserosal $(S S)$ layer $(n=20)$. b Immunohistochemistry for Ki67-positive area in the SM layer, MP, and SS layer of cancer tissue $(n=20)$. Scale bar $50 \mu \mathrm{m}$. c Growth curves of HSC44PE cells stimulated with HSC44PE-submucosal gastric fibroblast $(S M F)$ medium,

Dulbecco's modified Eagle's medium (DMEM)-SMF medium, HSC44PE-

subperitoneal gastric fibroblast $(S P F)$ medium, or DMEM-SPF medium. Three independent experiments were performed in triplicate
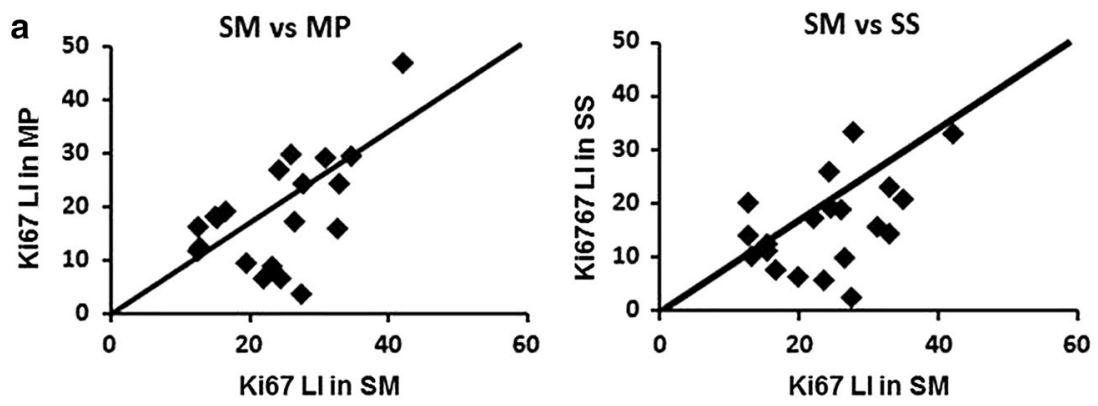

b

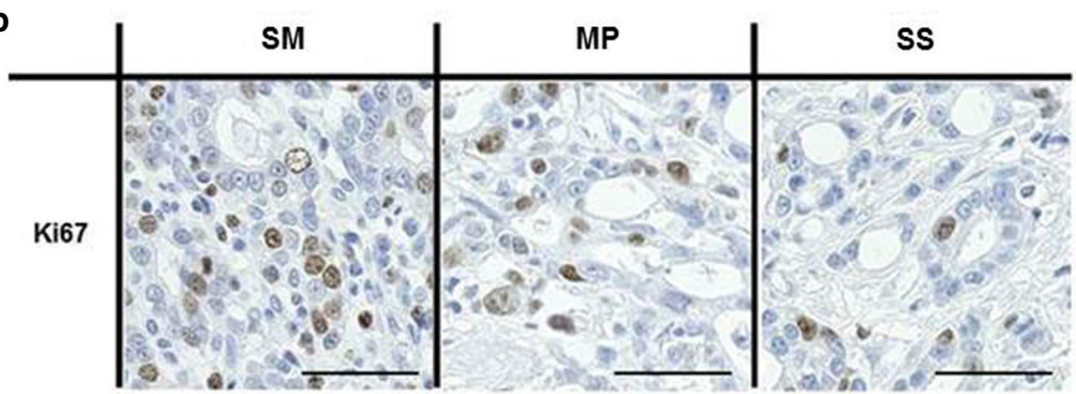

C

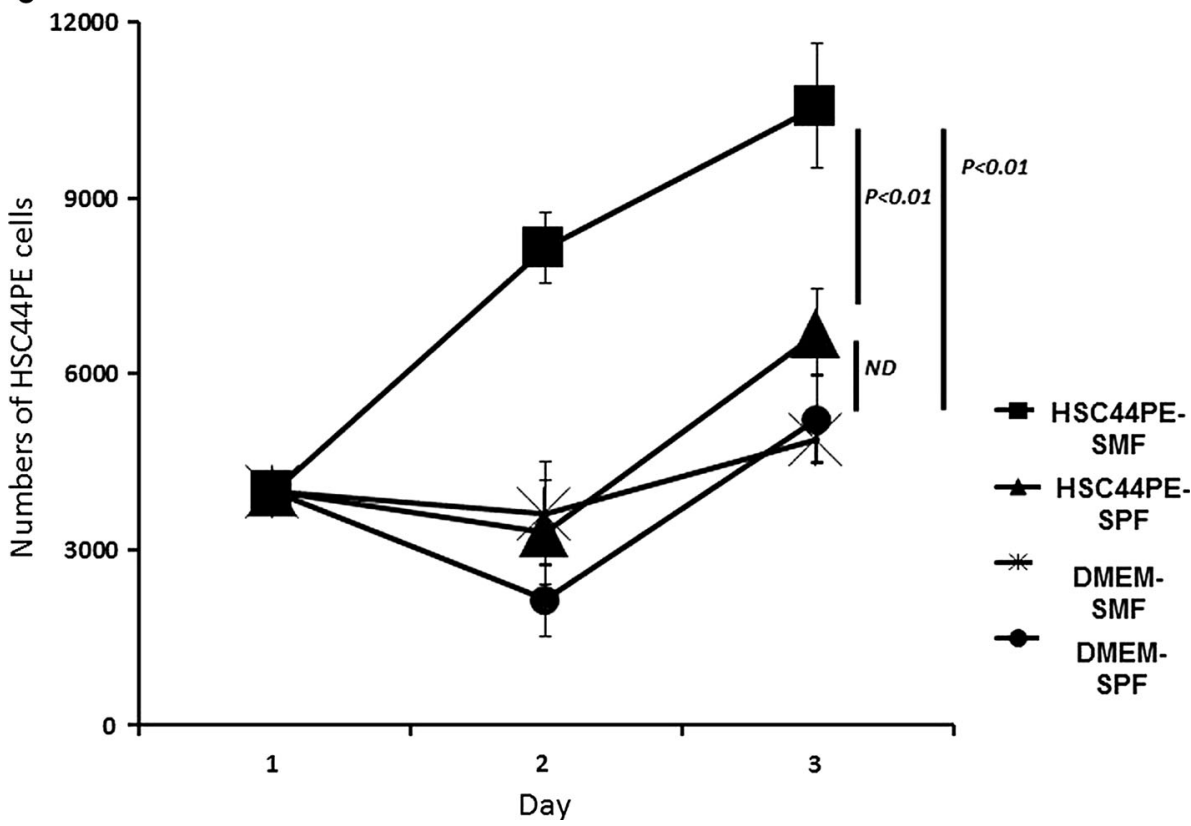

microenvironment in the submucosal layer was conducive for cancer cell growth.

Our results revealed that the number and percentage of M2 macrophages were significantly higher in the subserosal layer than in the submucosal layer (Fig. 2c, d). To examine whether the HSC44PE-SPF medium could induce the differentiation of monocytes into M2-like macrophages, we stimulated THP1 cells and examined the presence of M2 macrophage markers (high expression levels, arginase 1, IL10; low expression levels, IL-12A, IL-12B) [23] with use of qRT-PCR. Expression of ARG1 and ILIO was significantly higher in the THP1 cells treated with the HSC44PE-SPF medium than in the THP1 cells treated with the HSC44PESMF medium (Fig. 5a); on the other hand, expression of $I L 12 A$ and $I L 12 B$ was significantly lower in the THP1 cells treated with the HSC44PE-SPF medium (Fig. 5a). Thus, the HSC44PE-SPF medium promoted the differentiation of monocytes into M2-like macrophages.

The number of lymphocytes was higher in the submucosal layer than in the subserosal layer (Fig. 1). To examine whether SMFs more strongly than SPFs induced the migration of $\mathrm{T}$ cells, we examined the migration of Jurkat cells. Compared with the HSC44PE-SPF medium, the HSC44PE-SMF medium induced significantly more 
a
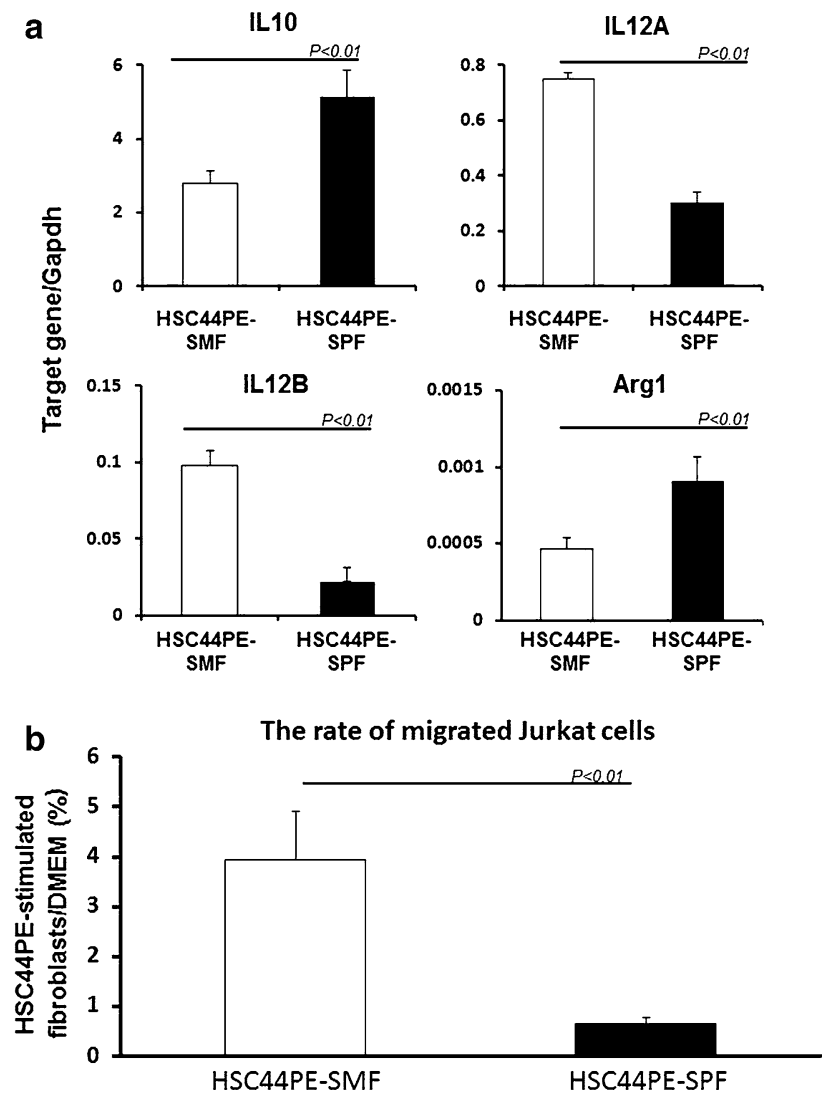

Fig. 5 Effects of fibroblasts stimulated with HSC44PE medium on the differentiation and migration of immune cells. a Differentiation of THP1 cells to M2-like macrophages following stimulation with HSC44PE-submucosal gastric fibroblast $(S M F)$ medium or HSC44PE-subperitoneal gastric fibroblast $(S P F)$ medium. The messenger RNA expression levels of IL10,IL12A,IL12B, and ARG1 in THP1 cells stimulated with HSC44PE-SMF medium or HSC44PESPF medium are shown. Three independent experiments were performed in triplicate. b Rate of migration of Jurkat cells following stimulation with HSC44PE-SMF medium or HSC44PE-SPF medium. The rate of Jurkat cell migration was determined by the number of migrating Jurkat cells after stimulation with HSC44PE-SPF medium or HSC44PE-SMF medium to the number of migrating Jurkat cells after stimulation with Dulbecco's modified Eagle's medium (DMEM). Three independent experiments were performed in triplicate

pronounced migration of Jurkat cells (Fig. 5b). This finding indicates that SMFs also have the ability to promote the migration of $\mathrm{T}$ lymphocytes, as observed by histopathology examinations.

\section{Discussion}

Our present study showed that the inflammatory environment varied from the early stage of inflammation to the late stage of inflammation according to the depth of cancer cell invasion. Site-specific fibroblasts might be involved in the regulation of site-specific inflammatory niche formation. The activation of site-specific fibroblasts by gastric-cancercell-conditioned medium showed significantly different inflammatory pathways between SMFs and SPFs.

In this study, we used two types of fibroblasts: SMFs SPFs. Histopathology examinations and analyses of gene expression indicated that the cancer microenvironment differed between the submucosal layer and the subserosal layer (Figs. 1, 2).

Many reports have suggested that the process of inflammation can be divided into two stages: early-stage inflammation and late-stage inflammation. During earlystage inflammation, platelet aggregation is followed by infiltration of neutrophils and leukocytes; these cells produce proinflammatory cytokines such as IL-1 $\beta$, IL-6, and TNF, which amplify the inflammatory response [24]. During late-stage inflammation, activated fibroblasts and M2 macrophages play major roles and produce TGF- $\beta$ and IL-10 to repair the tissue and suppress immune cell activity [24]. On the basis of these reports and the formation of cancer-associated inflammatory niches, the submucosal layer showed early-stage inflammation and the subserosal layer showed late-stage inflammation in this study (Figs. 1, $2,6)$. Thus, cancer-associated inflammation varies from early-stage inflammation to late-stage inflammation according to the depth of cancer cell invasion.

Fibroblasts are the most abundant stromal cells in cancer tissues. From the perspective of the kinetics of immune cells, chemokine (C-C motif) ligand 13 , TNF superfamily member 15 , and IL- $1 \beta$ promote infiltration and the accumulation of $\mathrm{T}$ lymphocytes at sites of inflammation [7]. HSC44PE-stimulated SMFs induced the migration of Jurkat cells and showed increased expression of CCL13, TNFSF 15, and ILIB (Figs. 3, 5b). These results indicate that cancer-stimulated SMFs promote the migration of lymphocytes. On the other hand, colony stimulating factor 1 , chemokine $(\mathrm{C}-\mathrm{C}$ motif) ligand 8 , chemokine $(\mathrm{C}-\mathrm{X}-$ $\mathrm{C}$ motif) ligand 5, and transforming growth factor $\beta_{2}$ are involved in the differentiation of M2 macrophages and promotion of fibrosis [25-28]. HSC44PE-stimulated SPFs induced the differentiation of THP1 cells into M2-like macrophages and showed increased expression of CSF1, CCL8, CXCL5, and TGFB2 (Figs. 3, 5a). These results suggest that SPFs promote the differentiation of monocytes into M2-like macrophages. Tumors with myofibroblast depletion have been reported to show a significant decrease in the overall peritumoral infiltration by $\mathrm{CD} 45^{+}$cells, $\mathrm{CD}^{+}$cells, and $\mathrm{CD} 19^{+} \mathrm{B}$ cells in pancreatic cancer [16]. The existence of fibroblasts would be necessary for the formation of an inflammatory niche, and each type of fibroblast has its own role in promoting the differentiation or migration of immune cells. 
In addition, the percentage of Ki67-positive cancer cells and the proliferation of HSC44PE cells were significantly higher in the submucosal layer containing SMFs (Fig. 4). A previous report also clarified that the major pool of proliferating cancer cells resided in the superficial layers (mucosa and submucosa) of gastric cancer [20]. Moreover, HSC44PE-stimulated SMFs showed enhanced expression of $I L 1 B, T N F S F 15$, and $C X C L 13$, which promote cancer cell growth (Fig. 3). On the other hand, HSC44PE-stimulated SPFs showed enhanced expressions of TGFB2 (Fig. 3). A previous report suggested that transforming growth factor $\beta_{1}$ effectively diminished the expression of the epithelial marker E-cadherin and enhanced the expression of the mesenchymal marker vimentin in gastric cancer cells [29]. These reports and results suggest that SMFs promote cancer cell growth and that SPFs promote cancer cell invasion.

In gastric cancer, cancer cell invasion occurs from the submucosal layer to the subserosal layer, progressing over time. Therefore, the submucosal layer would be expected to show late-stage inflammation and the subserosal layer would be expected to show early-stage inflammation. However, we obtained precisely the opposite result (Fig. 6). Thus, the responses of SPFs may differ according to the type of stimulation, whereas SMFs may respond similarly to both pathogens and cancers. In the case of sepsis, microorganisms react with inflammatory cells and fibroblasts in the peritoneal membrane, which results in the production of a large array of cytokines and chemokines [30]. Furthermore, it has been shown that following the intraperitoneal administration of lipopolysaccharide to mice, the cells lining the peritoneal membrane produce IL6 , IL-1 $\beta$, and TNF- $\alpha[31,32]$. These reports suggested that SPFs also produce the proinflammatory cytokines IL-1 $\beta$, IL-6, and TNF- $\alpha$. However, in our present experiment, stimulation with HSC44PE-conditioned medium did not induce the gene expression of proinflammatory cytokines in the SPFs (Fig. 3). Thus, the roles of SPFs might differ in infection and cancer.

Functional differentiation between SMFs and SPFs against cancer cells may depend on the external environment. The mucosae of the digestive tract provide an interface for interactions between multicellular hosts and their external environment [33]. Because of continuous environmental exposure, the mucosal surface is a major route for the entry of numerous pathogens [33]. The gastrointestinal tract contains gut-associated lymphoid tissues and mucosa-associated lymphoid tissues to protect against invasion by pathogens [34]. Both cancer cells and pathogens share the ability to destroy tissue during the inflammatory stage. From the standpoint of fibroblasts during inflammation, SMFs may acquire the ability to defend against the establishment of cancer cells as a result of

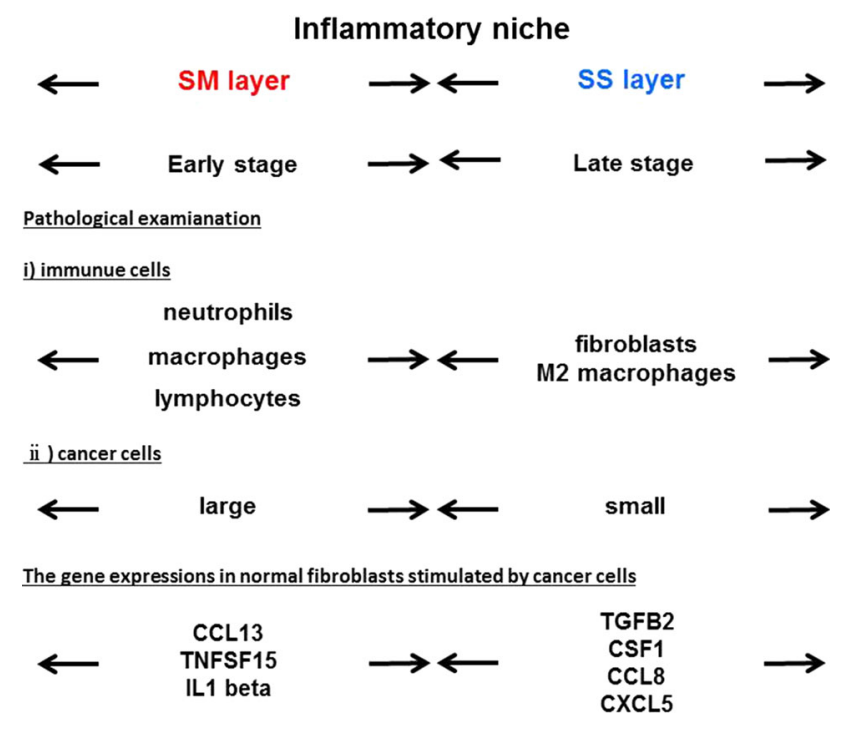

Fig. 6 Inflammatory niche formation in gastric cancer tissue. SM, SS

exposure to the threat of tissue destruction by pathogens. On the other hand, the serosa has a lower chance of being invaded by the numerous pathogens, resulting in the SPFs in the subserosal layer not having acquired the ability to attack such pathogens. Therefore, the responses of SMFs and SPFs to cancer cells may differ.

We conclude that the dynamic state of immune cells differs between the submucosal layer and the subserosal layer in cases of cancer. Site-specific fibroblasts regulate the formation of site-specific inflammatory niches in cancer tissues according to the depth of cancer cell invasion.

Acknowledgments We thank Yoshiko Onuma and Motoko Suzaki for helpful advice.

\section{Compliance with ethical standards}

Conflicts of interest The authors declare that they have no conflict of interest.

Ethical standards All procedures followed were in accordance with the ethical standards of the responsible committee on human experimentation (institutional and national) and with the Helsinki Declaration of 1964 and later versions.

\section{References}

1. Patel R, Filer A, Barone F, et al. Stroma: fertile soil for inflammation. Best Pract Res Clin Rheumatol. 2014;28:565-76.

2. Öhlund D, Elyada E, Tuveson D. Fibroblast heterogeneity in the cancer wound. J Exp Med. 2014;211:1503-23.

3. Kojima M, Higuchi Y, Yokota M, et al. Human subperitoneal fibroblast and cancer cell interaction creates microenvironment that enhances tumor progression and metastasis. PLOS ONE. 2014;9:e88018. 
4. Landskron G, De la Fuente M, Thuwajit P, et al. Chronic inflammation and cytokines in the tumor microenvironment. J Immunol Res. 2014;2014:149185.

5. Balkwill F, Mantovani A. Inflammation and cancer: back to Virchow? Lancet. 2001;357:539-45.

6. Candido J, Hagemann T. Cancer-related inflammation. J Clin Immunol. 2013;33:S79-84.

7. Coussens LM, Werb Z. Inflammation and cancer. Nature. 2002;420:860-7.

8. Colotta F, Allavena P, Sica A, et al. Cancer-related inflammation, the seventh hallmark of cancer: links to genetic instability. Carcinogenesis. 2009;30:1073-81.

9. Choi B, Lee EJ, Park YS, et al. Pentraxin-3 silencing suppresses gastric cancer-related inflammation by inhibiting chemotactic migration of macrophages. Anticancer Res. 2015;35:2663-8.

10. Wang F, Meng W, Wang B, et al. Helicobacter pylori-induced gastric inflammation and gastric cancer. Cancer Lett. 2014;345:196-202.

11. Szczepanik AM, Scislo L, Scully T, et al. IL-6 serum levels predict postoperative morbidity in gastric cancer patients. Gastric Cancer. 2011;14:266-73.

12. Chang WJ, Du Y, Zhao X, et al. Inflammation-related factors predicting prognosis of gastric cancer. World J Gastroenterol. 2014;20:4586-96.

13. Hodge DR, Hurt EM, Farrar WL. The role of IL-6 and STAT3 in inflammation and cancer. Eur J Cancer. 2005;41:2502-12.

14. Smyth MJ, Cretney E, Kershaw MH, et al. Cytokines in cancer immunity and immunotherapy. Immunol Rev. 2004;202:275-93.

15. McGettrick HM, Buckley CD, Filer A, et al. Stromal cells differentially regulate neutrophil and lymphocyte recruitment through the endothelium. Immunology. 2010;131:357-70.

16. Özdemir BC, Pentcheva-Hoang T, Carstens JL, et al. Depletion of carcinoma-associated fibroblasts and fibrosis induces immunosuppression and accelerates pancreas cancer with reduced survival. Cancer Cell. 2014;25:719-34.

17. Abe A, Kuwata T, Yamauchi C, et al. High mobility group box 1 (HMGB1) released from cancer cells induces the expression of pro-inflammatory cytokines in peritoneal fibroblasts. Pathol Int. 2014;64:267-75.

18. Yanagihara K, Tanaka H, Takigahira M, et al. Establishment of two cell lines from human gastric scirrhous carcinoma that possess the potential to metastasize spontaneously in nude mice. Cancer Sci. 2004;95:575-82.

19. Witowski J, Jorres A. Peritoneal cell culture: fibroblasts. Perit Dial Int. 2006;26:292-9.
20. Ramires M, David L, Leitão D, et al. Ki67 labelling index in gastric carcinomas. An immunohistochemical study using double staining for the evaluation of the proliferative activity of diffusetype carcinomas. J Pathol. 1997;182:62-7.

21. Ma YR, Ma YH. MIP- $1 \alpha$ enhances Jurkat cell transendothelial migration by up-regulating endothelial adhesion molecules VCAM-1 and ICAM-1. Leuk Res. 2014;38:1327-31.

22. Grivennikov SI, Greten FR, Karin M. Immunity, inflammation, and cancer. Cell. 2010;140:883-99.

23. Mantovani A, Sozzani S, Locati M, et al. Macrophage polarization: tumor-associated macrophages as a paradigm for polarized M2 mononuclear phagocytes. Trends Immunol. 2002;23:549-55.

24. Eming SA, Krieg T, Davidson JM. Inflammation in wound repair: molecular and cellular mechanisms. J Invest Dermatol. 2007;127:514-25.

25. Kim MS, Song HJ, Lee SH, et al. Comparative study of various growth factors and cytokines on type I collagen and hyaluronan production in human dermal fibroblasts. J Cosmet Dermatol. 2014;13:44-51.

26. Gaspar K, Kukova G, Bunemann E, et al. The chemokine receptor CCR3 participates in tissue remodeling during atopic skin inflammation. J Dermatol Sci. 2013;71:12-21.

27. Rousselle A, Qadri F, Leukel L, et al. CXCL5 limits macrophage foam cell formation in atherosclerosis. $J$ Clin Invest. 2013;123:1343-7.

28. Lee GT, Hong JH, Kwak C, et al. Effect of dominant negative transforming growth factor- $\beta$ receptor type II on cytotoxic activity of RAW 264.7, a murine macrophage cell line. Cancer Res. 2007;67:6717-24.

29. Zhang H, Liu L, Wang Y, et al. KLF8 involves in TGF-betainduced EMT and promotes invasion and migration in gastric cancer cells. J Cancer Res Clin Oncol. 2013;139:1033-42.

30. Heumann D, Glauser MP, Calandra T. Molecular basis of hostpathogen interaction in septic shock. Curr Opin Microbiol. 1998;1:49-55.

31. Stearns-Kurosawa DJ, Osuchowski MF, Valentine C, et al. The pathogenesis of sepsis. Annu Rev Pathol. 2011;6:19-48.

32. Tateda K, Matsumoto T, Miyazaki S, et al. Lipopolysaccharideinduced lethality and cytokine production in aged mice. Infect Immun. 1996;64:769-74.

33. Lamichhane A, Azegamia T, Kiyonoa H. The mucosal immune system for vaccine development. Vaccine. 2014;32:6711-23.

34. Kiyono H, Fukuyama S. NALT- versus Peyer's-patch-mediated mucosal immunity. Nat Rev Immunol. 2004;4:699-710. 